\title{
Distributed generation long-term planning in smart distribution systems considering daily optimal operation
}

\author{
Hatem Sindi* \\ Electrical and Computer Engineering Department, \\ University of Waterloo, \\ 200 University Ave W, \\ Waterloo, ON N2L 3G1, Canada \\ Email: hsindi@uwaterloo.ca \\ *Corresponding author
}

\section{Mostafa Shaaban}

Department of Electrical Engineering, American University of Sharjah, University City, P.O. Box 26666, Sharjah, UAE

Email: mshaaban@aus.edu

\section{Ehab El-Saadany}

Electrical and Computer Engineering Department, University of Waterloo, 200 University Ave W, Waterloo, ON N2L 3G1, Canada

Email: ehab@uwaterloo.ca

\begin{abstract}
An algorithm to maximise distributed generation (DG) hosting capacity in a system while lowering overall system cost is proposed in this paper. This algorithm considers several realistic aspects of long-term DG planning, such as cost effective design of feeder reinforcement. It allocates DGs and provide the type, size, location and year of installation. Both dispatchable and non-dispatchable DG technologies are used. The complexity of the problem necessitates modelling the problem as a mixed integer nonlinear programming. This is performed while considering the daily optimal operation of the allocated DGs. A case study of test system was conducted over a planning period of 20 years with every year consisting of eight-day patterns and each day having varied hours.
\end{abstract}

Keywords: planning; smart distribution system; optimisation; operation; smart grid; long-term.

Reference to this paper should be made as follows: Sindi, H., Shaaban, M. and El-Saadany, E. (2016) 'Distributed generation long-term planning in smart distribution systems considering daily optimal operation', Int. J. Process Systems Engineering, Vol. 4, No. 1, pp.19-40. 
Biographical notes: Hatem Sindi received his BSc from King Abdulaziz University, Jeddah, Saudi Arabia in 2007 and MASc from University of Waterloo, Waterloo, ON, Canada in 2013, both in Electrical Engineering. He is currently pursuing his $\mathrm{PhD}$ in the Department of Electrical and Computer Engineering, University of Waterloo, Waterloo, ON, Canada. His research interests include distribution system planning, reliability, renewable DG, enabling the electrification of transportation, storage systems, and smart grid.

Mostafa Shaaban received his BSc and MSc from Ain Shams University, Cairo, Egypt in 2004 and 2008, respectively, both in Electrical Engineering. In 2014, he received his $\mathrm{PhD}$ in Electrical Engineering from University of Waterloo, Waterloo, ON, Canada. His research interests include electrical machines, reliability, renewable DG, distribution system planning, electric vehicles, storage systems and smart grid.

Ehab El-Saadany received his BSc and MSc in Electrical Engineering from Ain Shams University, Cairo, Egypt in 1986 and 1990, respectively, and $\mathrm{PhD}$ in Electrical Engineering from the University of Waterloo, Waterloo, ON, Canada in 1998. Currently, he is a Professor in the Department of Electrical and Computer Engineering, University of Waterloo, Waterloo, ON, Canada. His research interests include smart grids operation and control, power quality, distributed generation, power electronics, digital signal processing applications to power systems, and mechatronics.

This paper is a revised and expanded version of a paper entitled 'Distributed generation long-term planning in smart distribution systems considering daily optimal operation' presented at 2015 IEEE International Conference on Smart Energy Grid Engineering (SEGE), Oshawa, ON, 17-19 August 2015.

\section{Introduction}

DG is of key interest as grids move to a smarter environment. Whether for grid-connected operation or isolated operation, microgrids must be planned properly, enabling higher private investment absorption and yielding a multi ownership system. In addition to environmental factors, DGs capacity maximisation in the system can provide economic benefits and take steps towards a smarter distribution system (resilient, reliable, self-healing, generation independent and operate in both islanded or grid connected modes of operation). Planning for future distribution systems requires deep understanding of technologies and players that are being introduced and integrated into the system. Although planning studies have existed as long as distribution systems, they are not becoming any easier than they are now. More challenges have risen recently with the rise of new paradigms causing planners to become more reactive than active (You et al., 2014). However, it is anticipated that practice will overcome the difficulties to accept new and complex planning frameworks as the planning problem becomes more complex (Pilo et al., 2012). For that, new planning frameworks that incorporate new concepts and envision the future process are needed. An example of these concepts is the active distribution network concept. This concept poses several challenges to planners, such as the future regulatory and technical aspects of integrating the active players in the system. Authors in Pilo et al. (2012) have identified three planning tools that need to be fulfilled: stochastic techniques, multi-objective planning and operational details of the system. 
Numerous research articles have tackled the operational details of distributed resources. These studies confirm that the operational details of distributed generations (DGs) can significantly affect the size and placement of the installed unit. For instance, the optimal integration of wind-based generators into the system considering ramping effects is studied in Xiang et al. (2013). Moreover, work performed in Menniti et al. (2012) and Bollen et al. (2012) examines the maximum installed capacity (hosting capacity) and their effects on power quality (voltage). In addition, in Marra et al. (2012), storage concepts are proposed to best allocate them based on voltage sensitivity analysis. Phase unbalancing condition caused by the integration of small single phase units (for e.g., microFITs) was studied in Mostafa et al. (2013). For power curtailment, Liew and Strbac (2002) studied the benefits of optimally operating installed DGs; the outcomes show two main benefits; voltage support at peak loading and minimum power curtailment at minimum loading can be achieved yielding greater hosting capacity. The work used linear programming (LP) based optimal power flow (OPF) on single year period. While sufficient for the purpose of validation, allocation and sizing problems require a more complex representation of loads and DGs and a suitable algorithm to solve the resulting mixed integer (linear or nonlinear) problem over a longer time span. However, the operational details attaining DGs (any technology) power curtailment while satisfying technical constraints in a long-term planning framework is still needed. This need is seen from two perspectives:

1 local distribution company (LDC), distribution company (DISCO) or technical virtual power plant (TVPP) perspective: the entity that holds the technical overview of the system and ensures adequate resources and efficient operation

2 producer/customer (consumer): usually referred to in literature as prosumers (Liang et al., 2014), they are mostly interested in the economic benefits of investment and, obviously, absorption of their investments in the first place.

LDC, DISCOs, or TVPP can offer incentives to private investors (or prosemers) to place distributed sources in optimal locations to improve the voltage profile and/or improve power quality and reliability of service as described in Celli et al. (2005). Moreover, in addition to political, regulatory and environmental drivers, accommodating rising loads and integrating renewable sources and emerging players in the system are added incentives to move to a smarter system (Djapic et al., 2007). This work aims to utilise emerging technologies in long-term planning of smart distribution systems. By employing the new technologies, operational details are factored in the planning procedure. Section 2 analyses the relationship between involved (existing and emerging) stakeholders. The problem is described then followed by formulation of the objective and constraints in Section 3. Section 4 describes the proposed architecture of solving the described planning problem.

\section{Stakeholders}

This section comprises three tasks: stakeholder identification and description, categorisation, and an investigation of the relationship between them. Description and identification are performed and stated based on key players in the smart distribution systems paradigm. The categorisation is then performed by means of an interest-influence 
matrix, where relationship assessment is made possible through the actor-linkage matrix. According to the National Institute of Standards and Technology (NIST), seven domains are suggested in their conceptual framework. These domains are used as a starting point for stakeholder identification and description in the following section.

Stakeholder analysis is commonly used for policy formulation and analysing complex and conflicting situations. It is important to mention that stakeholder analysis, especially in identification, is a subjective and iterative process. Some stakeholders that may be regarded as important and are associated with a key role in a system at first, may later become less or more involved. In Reed et al. (2009), three different rationales for performing stakeholder analysis are summarised, namely, descriptive, normative, and instrumental. Moreover, Reed et al. (2009) emphasises the fact that descriptive rationale is very rare for the mere sake of performing it, and is usually performed as a first step before normative- or instrument-based studies are conducted. This is because descriptive rationale involves only describing the relationship between a system and the players involved. It is important to mention that in the smart grid paradigm, instrumental rationale is important since it assumes a goal, and then analysis is conducted based on this goal. For normative studies, on the other hand, reaching a common goal is part of the analysis. Thus, it is first important to describe all stakeholders and their relation with the system (i.e., the smart grid). A summary of rationale, typology, and methods is adapted from Reed et al. (2009) and illustrated in Figure 1. However, only two methods can be performed by a practitioner/researcher: interest-influence and actor-linkage matrices.

Figure 1 Summary of rationale, typology, and methods used for stakeholder analysis

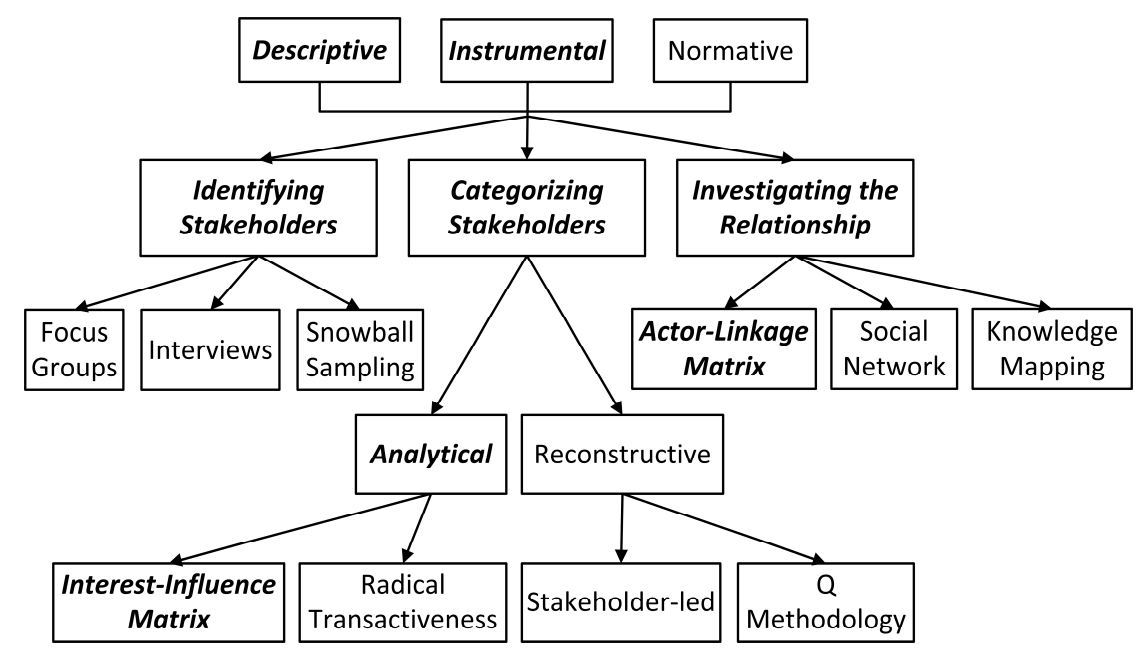

Stakeholders can have a significant impact on future smart distribution systems by the role they play. Stakeholders' roles in affecting the integrity and successful operation of the system are described. Moreover, stakeholders will be directly involved in envisioning the future distribution system. Although the NIST reports consensus among stakeholders with regard to interoperability standards, stakeholders are categorised differently with respect to interest and influence. This difference may be a reason for potential conflicts that arise in the long-term planning of smart distribution systems. Many stakeholders are expected to affect smart distribution systems with different degrees. The aforementioned 
domains they can be extended and placed as shown in Figure 2. It is clear from the figure that the most important category is the 'manage closely'. This category contains the most influential and the most interested stakeholders in a smart distribution system. They can provide key instruments to enable this transformation. For the 'keep satisfied' category, high influence can be made on the successful transformation to a smart distribution, but with low interest. Customers and the electrical equipment industry are important to monitor, as they affect the system, especially with the anticipated changing roles.

Figure 2 Stakeholder influence-interest matrix

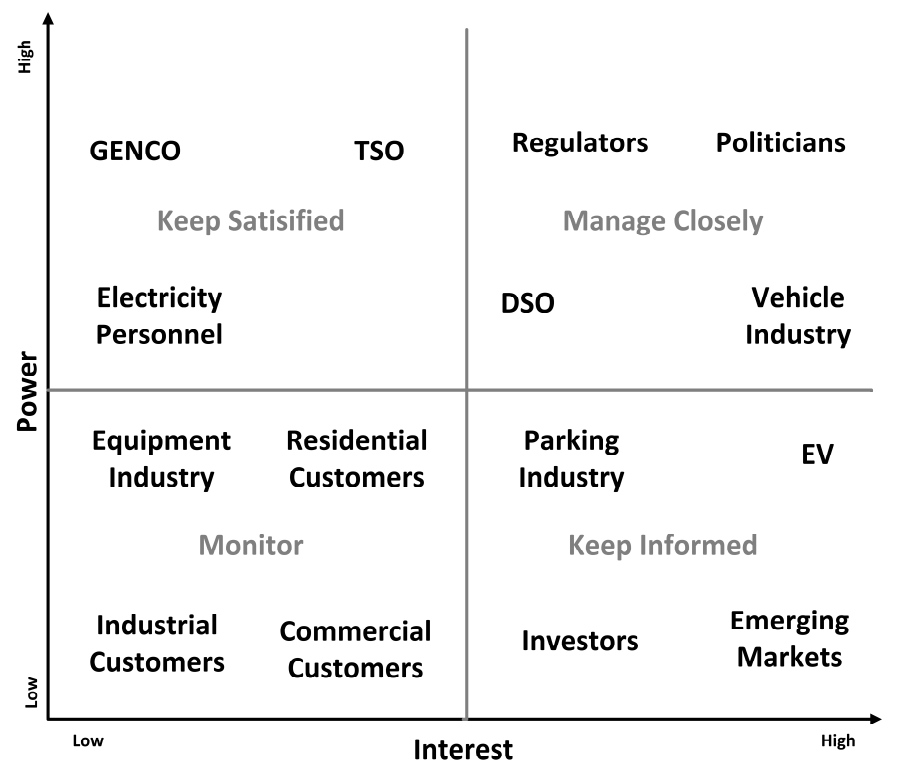

The 'keep informed' category poses the lowest challenges, as stakeholders in this category are already open to a smart distribution system. For a successful planning methodology, moving the 'keep satisfied' category toward a higher interest region is necessary. In addition, the 'monitor' category also needs to be moved to a higher interest region. These can be achieved by direct and strong communication in promoting the new concepts and advertising the benefits associated with such a transformation. The following section examines the communication and feedback levels between these categories. It is important to mention that this process is iterative and subject to changes according to the practitioner/researcher or time of the study.

Similar to the categorisation process, the relationship among stakeholders is also iterative. However, in this work, insights and conclusions about the relationship are drawn from surveys conducted in Ontario, Canada. The relation between the categories in general is summarised in Table 1. One of the important insights gained from this matrix is that increased feedback and communication between the 'keep informed' and 'monitor' categories are advised. In addition, since the 'keep informed' category is already highly interested in a new smart system, higher levels of communications and interactions are very advantageous with the 'keep satisfied' group. 
Table 1 Stakeholder actor-linkage matrix

\begin{tabular}{lcccc}
\hline Category & Monitor & Keep informed & Keep satisfied & Manage closely \\
\hline Monitor & - & $\mathrm{L}$ & $\mathrm{H}$ & $\mathrm{H}$ \\
Keep informed & $\mathrm{L}$ & - & $\mathrm{L}$ & $\mathrm{H}$ \\
Keep satisfied & $\mathrm{H}$ & $\mathrm{L}$ & - & $\mathrm{H}$ \\
Manage closely & $\mathrm{H}$ & $\mathrm{H}$ & $\mathrm{H}$ & - \\
\hline
\end{tabular}

Notes: L: none to low communication and feedback.

$\mathrm{H}$ : medium to high communication and feedback.

\section{Problem formulation}

The allocation and sizing of DGs in distribution systems is a critical problem, as they do not only affect the cost of upgrading the system, they may lead to catastrophic technical performance of the system if not properly designed. The problem can be divided into two major sub-problems:

- allocation, type, sizing and year of installation of DGs while satisfying physical and environmental constraints

- meeting technical constraints while minimising operational costs (OPF).

The first sub-problem introduces the mixed integer component to the overall problem due to the decisions regarding placement and the integer steps of sizing. For the second sub-problem, optimal power flow introduces high nonlinearity because of the power flow equations; in addition, the binary decision of upgrading lines causes an increase in the complexity of the overall problem. This problem, when studied for large systems and over long time spans, is very challenging computationally.

In a smart distribution system, conflicting interests are inevitable due to the various natures and roles of players. However, planning the distribution system while considering various interests may resolve some of the potential conflicts. For instance, absorption of private investment can be accommodated by enabling higher hosting capabilities in the system while benefiting the system operator [lowering paid incentives to DG owners (Celli et al., 2005)]. Therefore, description of all costs associated with all system players are presented.

\subsection{Objective function}

In trying to satisfy multiple players gains in a situation, social welfare maximisation is generally preferred. However, the distribution system is inherently incapable of maximising revenues since it is only made through selling eclectic power to end consumers. Therefore, the best practice is to minimise costs. On the other hand, for a regional planner, the interests of several stakeholders in distribution systems are in the core of any conducted study. For LDCs, minimising system upgrade requirements meeting technical and environmental constraints are needed. Investors' interests, on the other hand, are mainly focused on absorbing their investments while foreseeing acceptable returns. In order to comply with system requirements and engage investors, additional (not needed from LDC point of view) upgrades may be required. Therefore, 
the overall objective function that any regional planner interested in satisfying both parties is described in equations (1) and (3). This objective is subject to constraints formulated in equations (15) to (28).

$$
\begin{aligned}
\min \left(\mathrm{NPV}_{\text {Cost }}^{\text {System }}+\mathrm{NPV}_{\text {Cost }}^{\text {Investor }}\right) & \\
\mathrm{NPV}_{\text {Cost }}^{\text {System }}= & \mathrm{NPV}_{\text {Energy }}^{\text {Loss }}+\mathrm{NPV}_{\text {Energy }}^{\text {Unused }}+\mathrm{NPV}_{\mathrm{DG}}^{\text {APower }} \\
& +\mathrm{NPV}_{\text {Lines }}^{\text {Upgrade }}+\mathrm{NPV}_{\text {Grid }}^{\text {APower }}+\mathrm{NPV}_{\mathrm{DG}}^{\text {RPower }} \\
& +\mathrm{NPV}_{\text {Grid }}^{\text {RPower }} \\
\mathrm{NPV}_{\text {Cost }}^{\text {Investor }}= & \mathrm{NPV}_{\mathrm{DG}}^{\text {Capital }}+\mathrm{NPV}_{\mathrm{DG}}^{\mathrm{OM}}+\mathrm{NPV}_{\mathrm{DG}}^{\text {Fuel }}
\end{aligned}
$$

One can notice that in order to minimise overall costs, some system or investors costs may be increased compared to the case if one cost interest was the objective. For the regional planner, the increase in cost must be carefully studied and compared to base cases to reflect proper cost association to all parties. For instance, for $\mathrm{NPV}_{\text {Lines }}^{\text {Upgade }}$, $\mathrm{NPV}_{\mathrm{DG}}^{\text {Capital }}$ is strongly coupled and can either relax or pressure this cost. Therefore, further analysis on marginal contribution and benefit is required to assign the variation of costs. However, this formulation (i.e., considers both parties' interests) is considered successful if and only if the total cost compared to the case of single interest is lowered. Clear distinction can be drawn on the interests of the planners depending on the ownership of the units to be placed. For instance, it is allowed in some countries for the LDC to own the DG unit while not in others. However, the difference in ownership only directly affects two costs, namely $\mathrm{NPV}_{\mathrm{DG}}^{\mathrm{APower}}$ and $\mathrm{NPV}_{\mathrm{DG}}^{\mathrm{RPower}}$. In the case that the $\mathrm{LDC}$ owns the DG unit, it is counterintuitive to minimise such costs. Therefore, in this case, these two costs can be removed from equation (2). Nevertheless, it remains part of the overall cost endured as seen from the third party planner point of view in other cases. The following subsections describe the set of constraints and is followed by description of terms. The following list mathematical represents and describes each of the cost venues:

- Distributed generation capital cost: The first sub-problem seeks to optimally allocate and size the DG unit in a time that provides minimum capital cost. For every year, the amount of capital to be invested is calculated and summed over the study period according to 4 . It is necessary to mention that any algorithm optimising this cost must inherently favour larger units because of the \$/MVA rate at installation. Moreover, changes mostly involve increases, as the amount to be paid as net present value (NPV) decreases for larger units.

$$
\mathrm{NPV}_{\mathrm{DG}}^{\text {Capital }}=\sum_{y \in \mathcal{Y}} \frac{\sum_{i \in \mathcal{I}} \sum_{d g \in \mathcal{D} \mathcal{G}} C_{(i, d g, y)}^{\text {Capital }}}{(1+r)^{y}}
$$

- Cost of operation and maintenance of operating distributed generation units: an important detail of the aforementioned operational details required for an enhanced planning framework is the operation and maintenance costs associated with every $\mathrm{kW}$ operated. This cost can be represented in several forms such as $\$ / \mathrm{kW}$ or MW and $\$ / \mathrm{kWh}$ or MWh and calculated using equation (5). 
$\mathrm{NPV}_{\mathrm{DG}}^{\mathrm{OM}}=\sum_{y \in \mathcal{Y}} \frac{\sum_{i \in \mathcal{I}} \sum_{d \in \mathcal{D}} \sum_{h \in \mathcal{H}} \sum_{d g \in \mathcal{D} \mathcal{G}} P_{(i, d g, h, d, y)} \times C_{(i, d g, y)}^{O M}}{(1+r)^{y}}$

- $\quad$ Cost of fuel used in distributed generation units: Depending on options and planning area infrastructure, several fuel-based DG technologies can be considered. It is always beneficial to consider less environmentally harmful technologies, such as gas or biomass. However, the operational cost associated with any fuel-based DG can be calculated using equation (6).

$$
\mathrm{NPV}_{\mathrm{DG}}^{\text {Fuel }}=\sum_{y \in \mathcal{Y}} \frac{\sum_{i \in \mathcal{I}} \sum_{d \in \mathcal{D}} \sum_{h \in \mathcal{H}} \sum_{d g \in \mathcal{D} \mathcal{G}} P_{(i, d g, h, d, y)} \times C_{(i, d g, h, d, y)}^{\text {Fuel }}}{(1+r)^{y}}
$$

- Cost of active energy purchased from distributed generation: According to the Independent Electricity System Operator (2015a), the cost of purchasing active energy from distributed generation is usually regulated. Therefore, as part of the operational detail, minimising this cost for the overall benefit of the smart system is needed. In equation (7), the mathematical representation of such cost is illustrated. It is important to mention that some of the allocated DGs can be used for ancillary services and must be compensated for any reactive power or any other type of ancillary service provided.

$$
\mathrm{NPV}_{\mathrm{DG}}^{\mathrm{APower}}=\sum_{y \in \mathcal{Y}} \frac{\sum_{i \in \mathcal{I}} \sum_{d \in \mathcal{D}} \sum_{h \in \mathcal{H}} \sum_{d g \in \mathcal{D} \mathcal{G}} P_{(i, d g, h, d, y)} \times C_{(i, d g, h, d, y)}^{\mathrm{APower}}}{(1+r)^{y}}
$$

- Cost of reactive power purchased from distributed generation: It is important to mention that some of the allocated DGs can be used for ancillary services and must be compensated for any reactive power or any other type of ancillary service provided. Therefore, in equation (8), the mathematical representation of this cost is illustrated.

$$
\mathrm{NPV}_{\mathrm{DG}}^{\mathrm{RPower}}=\sum_{y \in \mathcal{Y}} \frac{\sum_{i \in \mathcal{I}} \sum_{d \in \mathcal{D}} \sum_{h \in \mathcal{H}} \sum_{d g \in \mathcal{D} \mathcal{G}} Q_{(i, d g, h, d, y)} \times C_{(i, d g, h, d, y)}^{\mathrm{RPower}}}{(1+r)^{y}}
$$

- Cost of active power purchased from grid: Unlike cost associated with DG purchased energy, energy purchased from the grid usually follows variable prices. Depending on the independent system operator and regulations, different market structures exist. However, these prices usually reflect market clearing prices of bulk electrical energy. In equation (9), the NPV of the energy purchased from the grid is outlined.

$$
\mathrm{NPV}_{\text {Grid }}^{\mathrm{APower}}=\sum_{y \in \mathcal{Y}} \frac{\sum_{d \in \mathcal{D}} \sum_{h \in \mathcal{H}} \sum_{i \in \mathcal{G}} P_{g(i, h, d, y)} \times C_{(i, h, d, y)}^{\mathrm{APower}}}{(1+r)^{y}}
$$


- Cost of reactive power purchased from grid: Reactive power support is essential to any optimal operation of a distribution system. In addition to demand, reactive power support is a common practice for voltage and power quality optimal operation. Equation (10) describes the NPV of the total reactive power imported from grid.

$$
\mathrm{NPV}_{\text {Grid }}^{\text {RPower }}=\sum_{y \in \mathcal{Y}} \frac{\sum_{d \in \mathcal{D}} \sum_{h \in \mathcal{H}} \sum_{i \in \mathcal{G}} P_{g(i, h, d, y)} \times C_{(i, h, d, y)}^{\mathrm{RPower}}}{(1+r)^{y}}
$$

- Cost of system active power losses: Minimising system costs associated with losses (both active and reactive) is directly related to better performance. Thus, equation (11) describes the associated NPV of active power losses endured in the system.

$$
\mathrm{NPV}_{\mathrm{Loss}}^{\mathrm{APower}}=\sum_{y \in \mathcal{Y}} \frac{\sum_{d \in \mathcal{D}} \sum_{h \in \mathcal{H}} \sum_{i \in \mathcal{I}} P_{g(i, h, d, y)}{ }_{(\text {loss })} \times C_{(i, h, d, y)}^{\mathrm{APower}}{ }_{\text {Loss }}}{(1+r)^{y}}
$$

- Cost of system reactive power losses: In equation (12), description of NPV associated with reactive power losses in the system is presented. Minimising this cost, although might be relatively small, directly affect system performance.

$$
\mathrm{NPV}_{\text {Loss }}^{\mathrm{RPower}}=\sum_{y \in \mathcal{Y}} \frac{\sum_{d \in \mathcal{D}} \sum_{h \in \mathcal{H}} \sum_{i \in \mathcal{I}} Q_{(i, h, d, y)}{ }_{(\text {loss })} \times C_{(i, h, d, y)}^{\mathrm{RPower}}{ }_{\text {Loss }}}{(1+r)^{y}}
$$

- Cost of surplus (unused) energy from distributed generation: Equation (13) shows the NPV of cost associated with wasted available resources (i.e., curtailed) as part of optimal operation dispatch.

$$
\mathrm{NPV}_{\text {Energy }}^{\text {Unused }}=\sum_{y \in \mathcal{Y}} \frac{\sum_{i \in \mathcal{I}} \sum_{d \in \mathcal{D}} \sum_{h \in \mathcal{H}} \sum_{d g \in \mathcal{D} \mathcal{G}} P_{(i, d g, h, d, y)}^{\mathrm{Unused}} \times C_{(i, d g, h, d, y)}^{\mathrm{Unused}}}{(1+r)^{y}}
$$

- Line upgrade cost As part of the overall system cost problem as seen in a system that considers all players' interests within this system, it is important to minimise the lines upgrade cost.

$$
\mathrm{NPV}_{\text {Lines }}^{\text {Upgrade }}=\sum_{l \in \mathcal{L}} \frac{C_{\mathrm{UP}_{(l)}}}{(1+r)^{y_{\mathrm{UP}(l)}}}
$$

\subsection{Constraints on distributed generation unit sizes and allocation}

These constraints are satisfied in the first step of the algorithm (genetic algorithm). It governs the placement and sizing of DG units:

$$
\begin{aligned}
& S_{(i, d g, y)}=\varpi_{(i, d g, y)} \times \kappa_{(i, d g, y)} \forall i \in \mathcal{Q}_{d g}, y \in \mathcal{Y} \\
& S_{(i, d g, y)} \leq S_{d g_{(\max )}} \forall i \in \mathcal{Q}_{d g}, y \in \mathcal{Y}
\end{aligned}
$$


where

$$
\begin{array}{r}
\varpi_{(i, d g, y) \quad \text { variable size of allocated DG of type } d g ;} \\
\varpi_{(i, d g, y)}:\left\{\begin{array}{l}
\text { Integer }: d g \text { only available in steps } \\
\text { Continuous : otherwise }
\end{array}\right. \\
\forall d g \in \mathcal{D G}
\end{array}
$$

\subsection{Power curtailment constraints}

With new technologies emerging and being introduced to power systems in general, new planning frameworks that take advantage of these technologies must be developed. For instance, wider communication capabilities allow for several components whom traditionally could not communicate with each other to freely and efficiently do. In addition, smart inverters allow for greater control on DGs output power. This additional formulation allows DGs to be allocated and then curtail their power if this allocation minimises the overall cost of the objective function. The following set of constraints utilises these capabilities to better plan future smart distribution systems.

$$
\begin{aligned}
S_{(i, d g, h, d, y)}^{i n j}= & \Psi_{(i, d g, h, d, y)} \times A F_{(d g, h, d, y)} \times S_{(i, d g, y)} \\
& \forall i \in \mathcal{I}, d g \in \mathcal{D} \mathcal{G}, h \in \mathcal{H}, d \in \mathcal{D}, y \in \mathcal{Y} \\
P_{(i, d g, h, d, y)} \geq & P F_{(i, d g, h, d, y)} \times S_{(i, d g, h, d, y)}^{i n j} \\
& \forall i \in \mathcal{I}, d g \in \mathcal{D} \mathcal{G}, h \in \mathcal{H}, d \in \mathcal{D}, y \in \mathcal{Y} \\
Q_{(i, d g, h, d, y)} \leq & \sin \left[\cos ^{-1}\left(P F_{(i, d g, h, d, y)}\right)\right] \times S_{(i, d g, h, d, y)}^{i n j} \\
& \forall i \in \mathcal{I}, d g \in \mathcal{D} \mathcal{G}, h \in \mathcal{H}, d \in \mathcal{D}, y \in \mathcal{Y} \\
S_{(i, d g, h, d, y)}^{i n j}= & \sqrt{P_{(i, d g, h, d, y)}^{2}+Q_{(i, d g, h, d, y)}^{2}} \\
& \forall i \in \mathcal{I}, d g \in \mathcal{D} \mathcal{G}, h \in \mathcal{H}, d \in \mathcal{D}, y \in \mathcal{Y}
\end{aligned}
$$

\subsection{Power flow constraints}

Although they add to the complexity of the problem, power flow constraints are essential to any optimal planning or operation study. Power flow equations can be represented in a linear fashion, but this algorithm deploys nonlinear power flow representation for accuracy.

$$
\begin{aligned}
& P_{g_{(i, h, d, y)}}+\sum_{d g \in \mathcal{D} \mathcal{G}} P_{(i, d g, h, d, y)}-P_{D(i, h, d, y)} \\
& =\sum_{j \in \mathcal{J}} V_{(i, h, d, y)} V_{(j, h, d, y)} Y_{(i, j, y)} \cos \left(\theta_{(i, j, y)}+\delta_{(j, h, d, y)}-\delta_{(i, h, d, y)}\right) \\
& \quad \forall i \in \mathcal{I}, h \in \mathcal{H}, d \in \mathcal{D}, y \in \mathcal{Y}
\end{aligned}
$$




$$
\begin{aligned}
& P_{g_{(i, h, d, y)}}=\left\{\begin{aligned}
P_{g_{(i, h, d, y)}}: & i \in \mathcal{G} \\
: & i \notin \mathcal{G}
\end{aligned}\right. \\
& \forall i \in \mathcal{I}, h \in \mathcal{H}, d \in \mathcal{D}, y \in \mathcal{Y} \text {; } \\
& P_{g}^{R e v} \leq P_{g_{(i, h, d, y)}} \leq P_{\text {Max }}^{g} \forall i \in \mathcal{G} \\
& Q_{g_{(i, h, d, y)}}+\sum_{d g \in \mathcal{D} \mathcal{G}} Q_{(i, d g, h, d, y)}-Q_{D(i, h, d, y)} \\
& =-\sum_{j \in \mathcal{J}} V_{(i, h, d, y)} V_{(j, h, d, y)} Y_{(i, j, y)} \cos \left(\theta_{(i, j, y)}+\delta_{(j, h, d, y)}-\delta_{(i, h, d, y)}\right) \\
& \forall i \in \mathcal{I}, h \in \mathcal{H}, d \in \mathcal{D}, y \in \mathcal{Y} \\
& Q_{g_{(i, h, d, y)}}=\left\{\begin{aligned}
Q_{g_{(i, h, d, y)}:} & i \in \mathcal{G} \\
: & i \notin \mathcal{G}
\end{aligned}\right. \\
& \forall i \in \mathcal{I}, h \in \mathcal{H}, d \in \mathcal{D}, y \in \mathcal{Y} \text {; } \\
& Q_{g}^{R e v} \leq Q_{g_{(i, h, d, y)}} \leq Q_{\text {Max }}^{g} \forall i \in \mathcal{G} ;
\end{aligned}
$$

\subsection{Voltage limits constraints}

$$
\begin{aligned}
& V_{\min }^{\mathrm{spec}} \leq V_{g_{(i, h, d, y)}} \leq V_{\max }^{\mathrm{spec}} \\
& \forall i \in \mathcal{I}, h \in \mathcal{H}, d \in \mathcal{D}, y \in \mathcal{Y}
\end{aligned}
$$

\subsection{Line upgrades and current constraints}

$$
\begin{aligned}
I_{(l, h, d, y)} \leq & I_{(l, h, d, y)_{\text {max }}} \\
\forall l \in \mathcal{L}, & h \in \mathcal{H}, d \in \mathcal{D}, y \in \mathcal{Y} \\
\mathrm{UP}_{(l)}= & \begin{cases}1: & I_{(l, h, d, y)} \geq I_{(l, y)_{\max }} \\
0: & I_{(l, h, d, y)}<I_{(l, y)_{\max }}\end{cases} \\
& \forall l \in \mathcal{L}, h \in \mathcal{H}, d \in \mathcal{D}, y \in \mathcal{Y} \\
I_{(l, y)_{\max }}= & \left\{\begin{array}{c}
I_{(l)}^{C A P}: \forall y<y_{\left(\mathrm{UP}_{(l)}\right)} \\
A_{(l)} \times I_{(l)}^{C A P}: \forall y \geq y_{\left(\mathrm{UP}_{(l)}\right)} \\
\forall l \in \mathcal{L}, y \in \mathcal{Y}
\end{array}\right.
\end{aligned}
$$

\section{Proposed solving algorithm}

Most population-based techniques do not guarantee global optimality. However, it is very common to find numerous variations of heuristic means used to solve long-term planning problems (Foster et al., 2014; Gandomkar and Tolabi, 2010; Kalantari and Kazemi, 2011; Sheng et al., 2015; Silvestri et al., 1999; Di Silvestre et al., 2014; Mori and Yoshida, 
2009). Figure 3 describes the flowchart of the proposed algorithm. It solves the first subproblem by means of a genetic algorithm. A typical gene would include type, size, location, and year of placement of each DG. This will yield cost described in equation (4). Then this generation is passed to the OPF engine, which in part solves the second subproblem and stores the operational costs described in equations (5) to (14). This sequence is subject to several technical constraints described in Section 3 and repeated until the exit criteria is met.

Figure 3 Algorithm flowchart

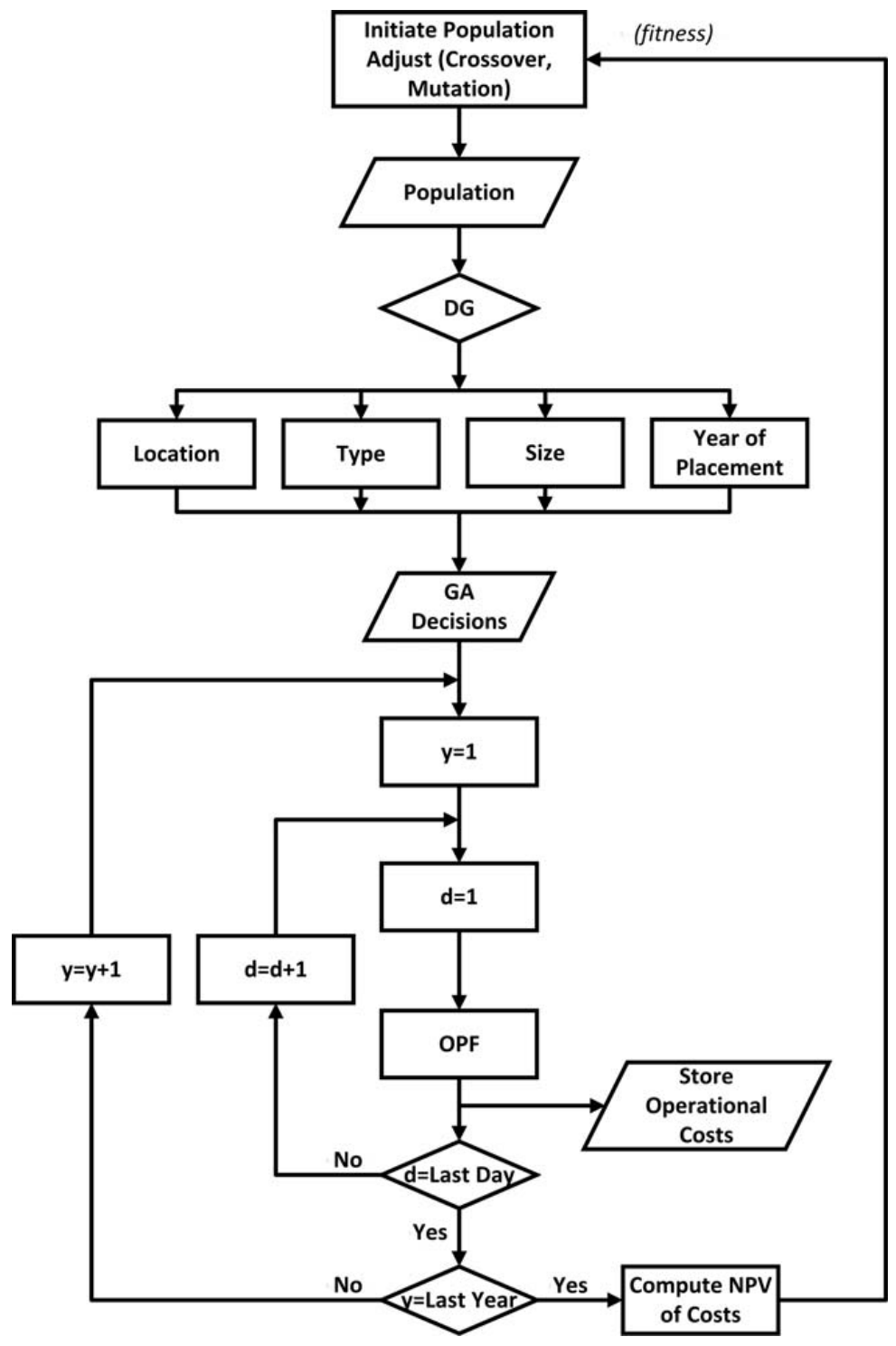


Figure 4 38-bus test system

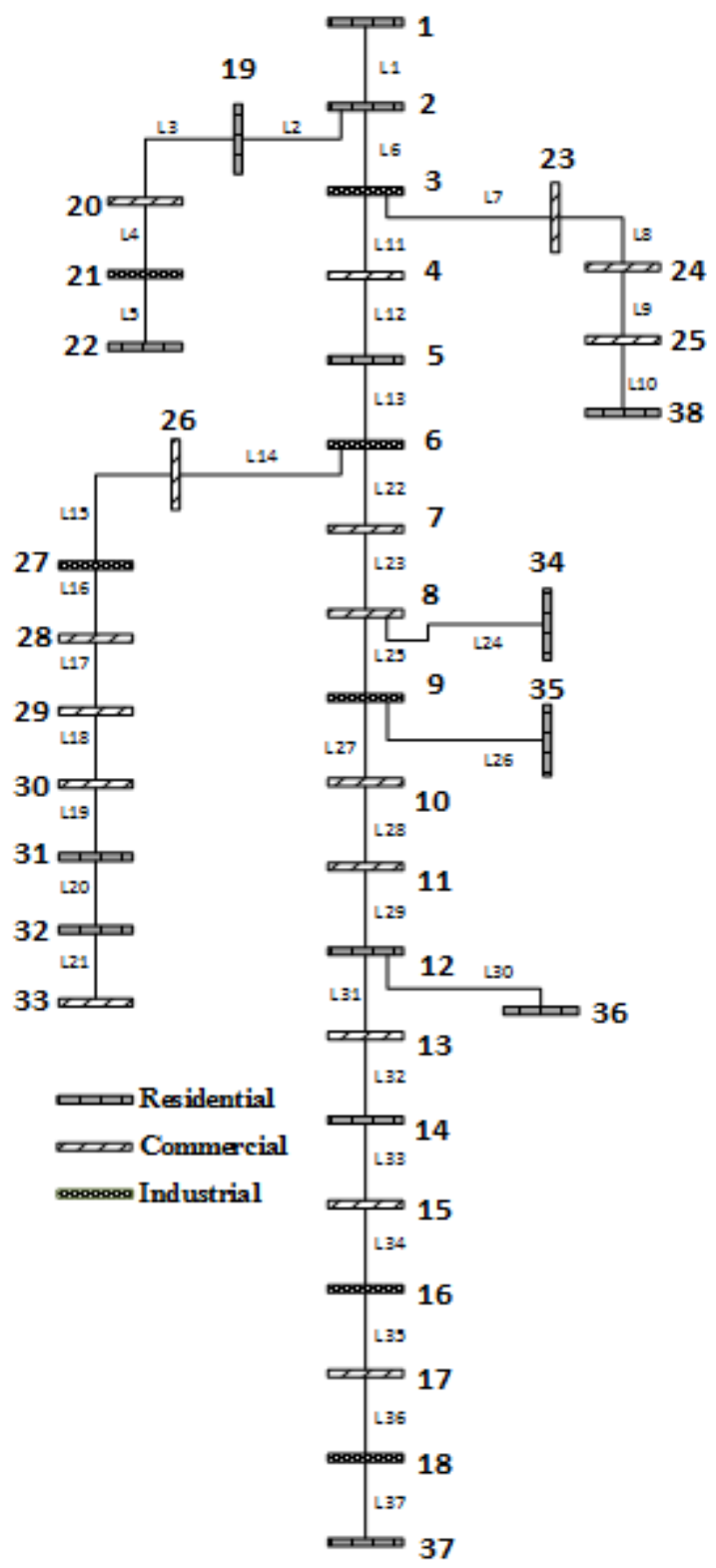

\section{Case study}

The daily variation is taken for both non-dispatchable generation and loads. Moreover, the loads also differ in type, namely, residential, commercial and industrial. This work also considers eight days to reflect seasonal and day type (weekday or weekend). For each season, two days reflecting a weekday and a weekend patterns are adapted from 
Hadley (1993). This allows the problem to be more dynamic. Therefore, the costs described in Section 3 are multiplied by $\frac{365}{8}$ to reflect the total days in a year. Results are compared using a planning methodology without consideration of the daily optimal operations [i.e., equations (17) to (20)]. Both are constrained according to most recent regulations and technical parameters. Moreover, the results clearly indicate a superiority of the proposed methodology, lower costs, lower average voltage deviations and higher DG hosting capacity. This case study is performed on a typical test system found in the literature (Singh et al., 2007). Renewable data used are adapted from Abdelaziz et al. (2014) and shown in Figure 7. The test system, shown in Figure 4, is composed of residential, commercial and industrial loads with different loading levels. These levels are illustrated in Figure 5.

Figure 5 A discretised day pattern of hourly loading variation for three customer types over a 24-hour span from $6 \mathrm{AM}$ to $6 \mathrm{AM}$

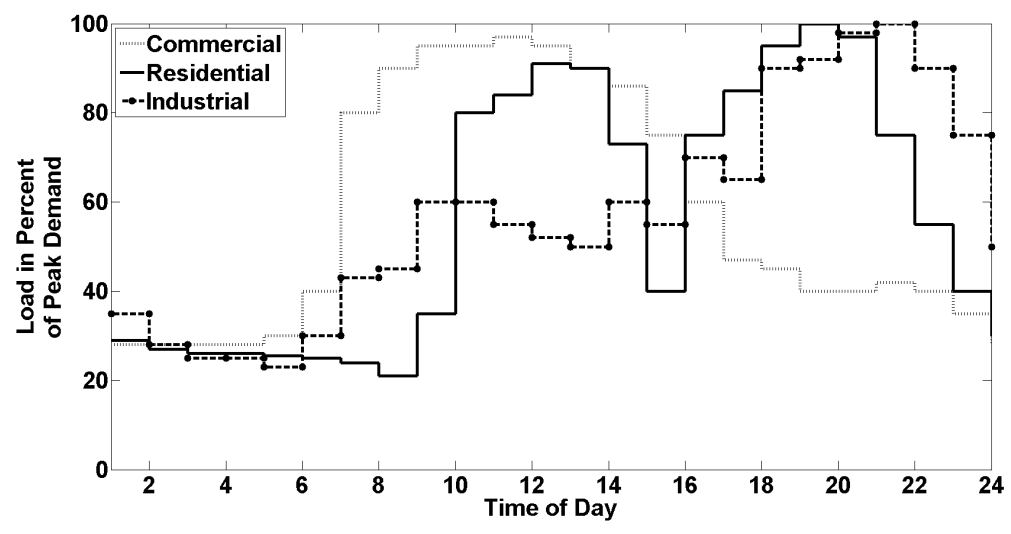

The objective to be minimised is shown in equation (1). The costs are adapted from Zidan et al. (2013), and the energy prices are based on the average hourly prices in Ontario over the past four years and retrieved from Independent Electricity System Operator (2015b). For reactive power, average compensation for reactive power support paid by Independent Electricity System Operator (2015b) in 2014 is used.

The study is performed for a period of 20 years with a load increase of $10 \%$ per year as seen in Figure 6.

Three types of DGs are considered: wind, solar and gas turbine. Hourly patterns of wind and solar power are illustrated in Figure 7.

However, in this case study, the cost of purchased energy from DGs is not considered. The reason for excluding this cost is to mimic the behaviour of a utility owned DGs where the energy is virtually free. Nevertheless, the cost of curtailed (unused) energy is minimised by associating a cost to it. The cost used in this work for curtailed power (depending on size and technology of DG) is adapted from Independent Electricity System Operator (2015a) (i.e., FIT and microFIt programs). Comparisons are made with base cases: a system with no DG allocation (only line upgrades) (i.e., Base 1) and a system with allocation of DGs neglecting the operational details (i.e., power curtailment, operation and maintenance, and fuel (i.e., Base 2). It is assumed in this case study that fuel costs are time and location independent, and that candidate buses are 
limited in the case of wind turbines due to the physical and environmental constraints and relaxed for the other two technologies considered in this case study (solar and gas-based).

Figure 6 Active and reactive peak demand over 20 years with a typical 10\% annual load rise in per unit (see online version for colours)

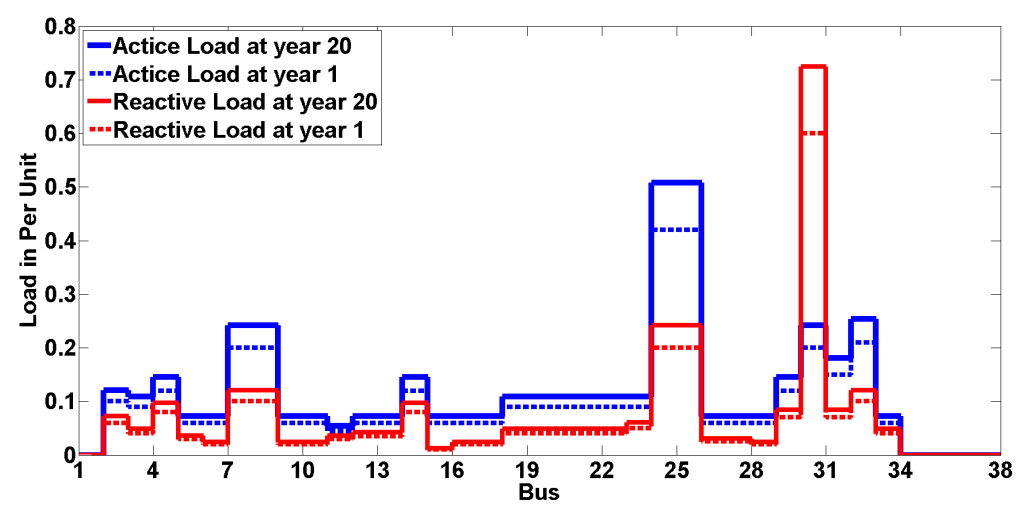

Figure 7 Discretised hourly available capacity of renewable power over a 24-hour span from $6 \mathrm{AM}$ to $6 \mathrm{AM}$

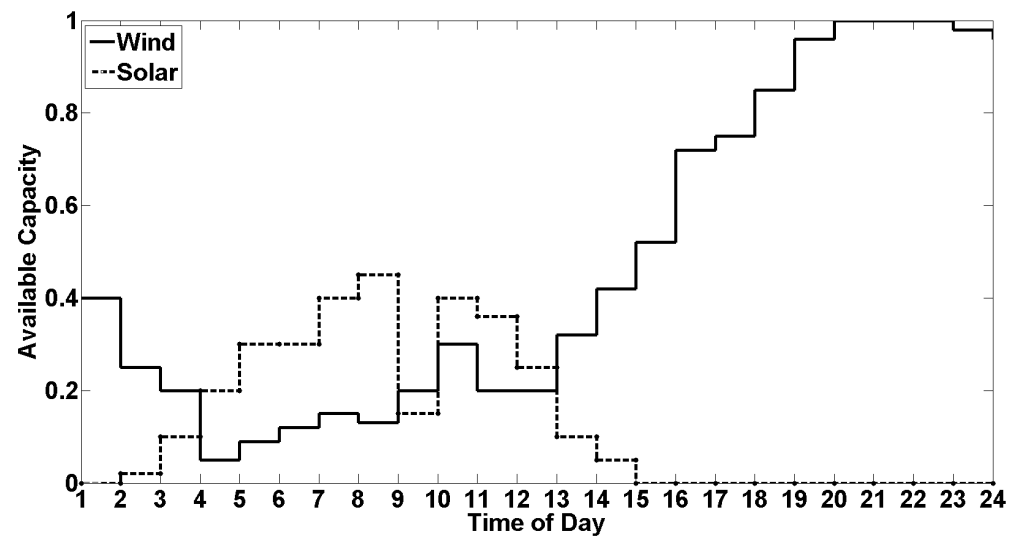

\section{Results and discussions}

It is clear from Table 2 that the proposed algorithm is superior to both Base 1 and Base 2 algorithms when it comes to total incurred costs. It is important to mention that total active power losses cost is slightly higher $(7.1 \%)$ in the proposed algorithm than Base 2 due to variation of time and price of the incurred losses, yet the total active losses in the system is $17 \%$ in the proposed scheme. The lower active losses yields as a byproduct better voltage profiles. In the case of Base 2, the average voltage deviation is lowered by $64.6 \%$ than Base 1. This improvement is very significant and improving it without leading to additional costs is achieved by the proposed algorithm. Additional $71.5 \%$ reduction in the average voltage deviation from Base 2 is achieved by the proposed 
methodology. This is almost $90 \%$ improvement if compared to Base 1. Using the proposed algorithm, higher absorption of investments was achieved. This can lead to a smarter, self-sustained, and resilient future distribution systems. About ten MVA of DG capacity were absorbed into the system while reducing the overall costs. The proposed methodology signifies the importance of higher private investment absorption in terms of better performance. This enhanced performance is key in defining a smart distribution system.

Table 2 Results of the case study comparing two base cases with the proposed algorithm

\begin{tabular}{lccccc}
\hline & Base 1 & Base 2 & $\%$ B2/B1 & Proposed & $\%$ Pr/B2 \\
\hline Total cost \$ & $369,969,184$ & $282,885,715$ & -23.5 & $258,507,940$ & -8.6 \\
Total return \$ & 0 & $87,083,469$ & 100.0 & $111,461,244$ & 28.0 \\
Active power (grid) \$ & $281,706,123$ & $135,256,035$ & -52.0 & $108,116,957$ & -20.1 \\
Active losses MW & 3.25904011 & 1.53979326 & -52.8 & 1.27736575 & -17.0 \\
Active losses \$ & 311,192 & 122,401 & -60.7 & 131,089 & 7.1 \\
Line upgrades \$ & $1,611,523$ & $5,216,186$ & 223.7 & $1,861,005$ & -64.3 \\
Reactive power (grid) \$ & $86,252,801$ & $67,290,457$ & -22.0 & $65,654,565$ & -2.4 \\
Reactive losses MVAR & 1.99991317 & 0.98335863 & -50.8 & 0.80240931 & -18.4 \\
Reactive losses \$ & 87,543 & 43,386 & -50.4 & 35,470 & -18.2 \\
Gas DG capital \$ & 0 & $15,040,328$ & 100.0 & $20,698,753$ & 37.6 \\
Solar DG capital \$ & 0 & $42,606,714$ & 100.0 & $46,702,933$ & 9.6 \\
Wind DG capital \$ & 0 & $6,763,230$ & 100.0 & $4,828,843$ & -28.6 \\
Gas \$ & 0 & $1,732,700$ & 100.0 & $1,958,563$ & 13.0 \\
Curtailed energy \$ & 0 & $8,609,527$ & 100.0 & $8,252,251$ & -4.1 \\
O\&M \$ & 0 & 204,745 & 100.0 & 267,506 & 30.7 \\
DG capacity MVA & 0 & 6.0403 & 100.0 & 10.0062 & 65.7 \\
Voltage deviation & 0.18742469 & 0.06629606 & -64.6 & 0.01891183 & -71.5 \\
\hline
\end{tabular}

Figure 8 Voltage profile containing minimum voltage occurring in the system in all three cases

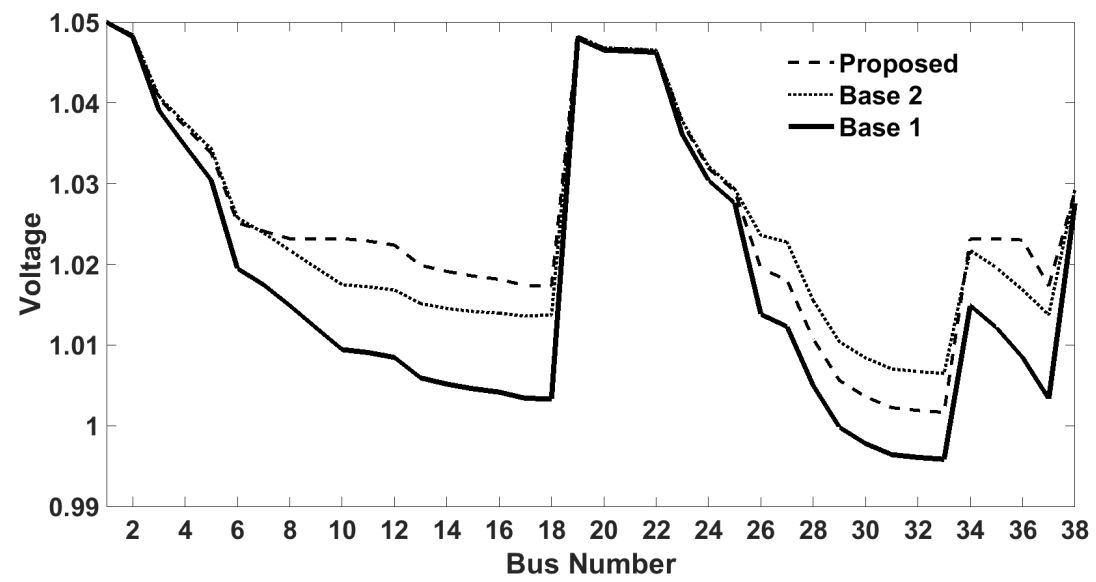


Distributed generation long-term planning in smart distribution systems

Table 3 Technology, size, location and year of allocated DGs using the proposed algorithm

\begin{tabular}{|c|c|c|c|c|c|c|c|}
\hline$i / y$ & 3 & 5 & 8 & 13 & 19 & 20 & $\sum_{d g \in \mathcal{D G}}$ \\
\hline 5 & & & & & & 0.1 & 0.1 \\
\hline 7 & & & & 0.1 & & & 0.1 \\
\hline 8 & & & 0.2 & & & & 0.2 \\
\hline 10 & & 1.2348 & & & & & 1.2348 \\
\hline 12 & & & 0.9 & & & & 0.9 \\
\hline 14 & & & & & & 0.8123 & 0.8123 \\
\hline 27 & & & & & & 1.5 & 1.5 \\
\hline 29 & & & 1.2421 & & & & 1.2421 \\
\hline 30 & & & & & 1.3 & 0.8 & 2.1 \\
\hline 32 & & & & & & 1.0842 & 1.0842 \\
\hline 36 & 0.1532 & & & & & & 0.1532 \\
\hline 38 & & & & & & 0.5796 & 0.5796 \\
\hline$\sum_{i \in \mathcal{I}}$ & 0.1532 & 1.2348 & 2.3421 & 0.1 & 1.3 & 4.8761 & 10.0062 \\
\hline
\end{tabular}

Table 4 Technology, size, location and year of allocated DGs using the methodology of base 2

\begin{tabular}{|c|c|c|c|c|c|c|c|c|c|c|c|c|}
\hline$i / y$ & 4 & 5 & 6 & 8 & 9 & 12 & 16 & 17 & 18 & 19 & 20 & $\sum_{d g \in \mathcal{D G}}$ \\
\hline 4 & & & & & & & & & & 0.23 & & 0.23 \\
\hline 12 & & & & & & & 0.31 & & & & & 0.33 \\
\hline 13 & & 0.17 & & & & & & & & & & 0.13 \\
\hline 14 & & & & & & & & & & 0.22 & 0.1 & 0.33 \\
\hline 15 & & & & & & & & 0.14 & & & & 0.14 \\
\hline 16 & & & & & 0.5 & & & & & & & 0.50 \\
\hline 17 & & & 0.31 & & & & & & & & & 0.31 \\
\hline 18 & 0.19 & & & & & & & & & & & 0.19 \\
\hline 22 & & & & 0.1 & & & & & & & & 0.10 \\
\hline 23 & & & & & & & 0.60 & & & 0.20 & & 0.80 \\
\hline 27 & 1.47 & & & & & & & & & & & 1.47 \\
\hline 29 & & & & & & 0.32 & & & & & & 0.32 \\
\hline 32 & & & & & & & & & & 0.20 & & 0.20 \\
\hline 33 & & & & & & & & 0.10 & & & & 0.10 \\
\hline 35 & & & & & & & & & 0.21 & & & 0.21 \\
\hline 38 & & & & & 0.7 & & & & & & & 0.70 \\
\hline$\sum_{i \in \mathcal{I}}$ & 1.66 & 0.17 & 0.31 & 0.1 & 1.2 & 0.32 & 0.91 & 0.24 & 0.21 & 0.85 & 0.1 & 6.04 \\
\hline
\end{tabular}


Figure 9 Average voltage profile occurring in the system in all three cases

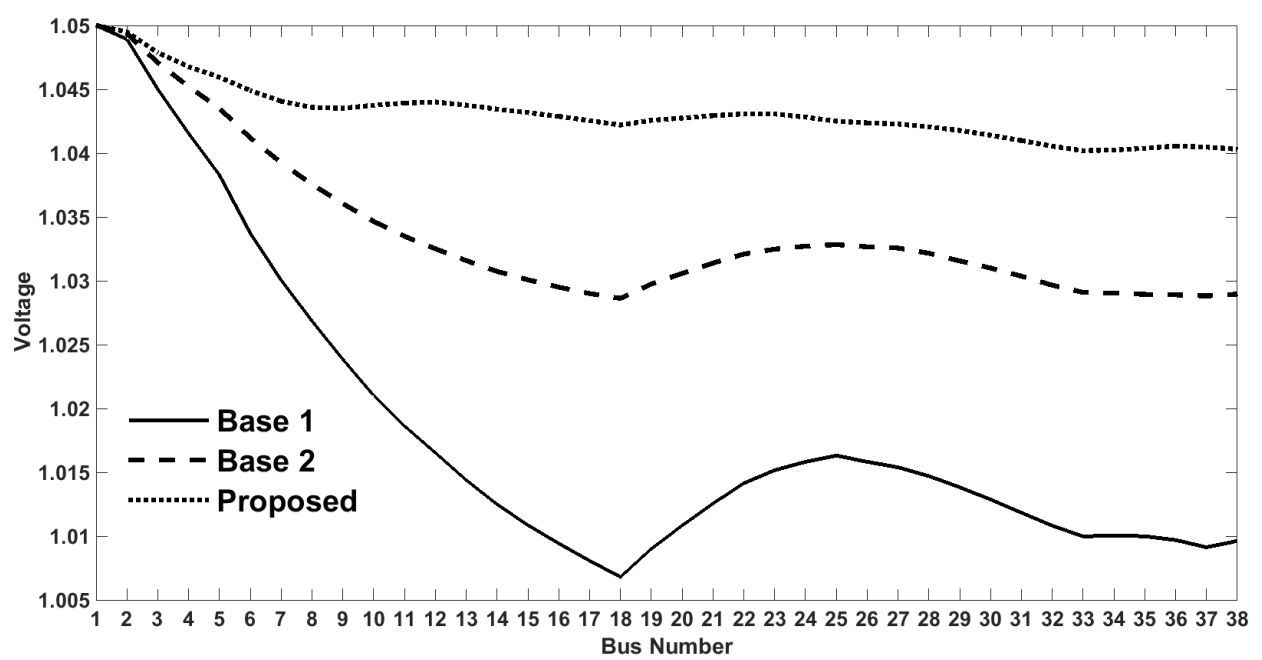

\section{Conclusions}

This work can be concluded in several points: a proposed algorithm to maximise DG hosting capacity, long-term planning incorporating hourly optimal power flow and proposed objective function yielding a new point of view that reflects interests of different stakeholders of distribution systems. In order to achieve these points, description of costs associated with the planning problem and formulation of objective and constraints are illustrated. Validation is performed through the means of a case study that reflected the superiority of the proposed planning algorithm. It is proposed in this work to whom this framework is directed (i.e., to regional planners). The party that conducts this planning should be independent of any conflict of interests and aims to maximise benefits to all system players (stakeholders). Further studies in the area of conflict and cooperation analysis is required for a successful transition the future paradigm.

\section{Acknowledgements}

Hatem Sindi would like to acknowledge King Abdulaziz University for sponsoring his $\mathrm{PhD}$ studies and research. 


\section{References}

Abdelaziz, M.M.A., Shaaban, M.F., Farag, H.E. and El-Saadany, E.F. (2014) 'A multistage centralized control scheme for islanded microgrids with PEVs', IEEE Transactions on Sustainable Energy, Vol. 5, No. 3, pp.927-937.

Bollen, M.H.J., Etherden, N., Yang, K. and Chang, G.W. (2012) 'Continuity of supply and voltage quality in the electricity network of the future', Proceedings of International Conference on Harmonics and Quality of Power, ICHQP, pp.375-377.

Celli, G., Ghiani, E., Mocci, S. and Pilo, F. (2005) 'A multiobjective evolutionary algorithm for the sizing and siting of distributed generation', IEEE Transactions on Power Systems, May, Vol. 20, No. 2, pp.750-757.

Di Silvestre, M.L., Graditi, G. and Sanseverino, E.R. (2014) 'A generalized framework for optimal sizing of distributed energy resources in micro-grids using an indicator-based swarm approach', IEEE Transactions on Industrial Informatics, Vol. 10, No. 1, pp.152-162.

Djapic, P., Ramsay, C., Pudjianto, D., Strbac, G., Mutale, J., Jenkins, N. and Allan, R. (2007) 'Taking an active approach', IEEE Power and Energy Magazine, Vol. 5, No. 4, pp.68-77.

Foster, J.D., Berry, A.M., Boland, N. and Waterer, H. (2014) 'Comparison of mixed-integer programming and genetic algorithm methods for distributed generation planning', IEEE Transactions on Power Systems, Vol. 29, No. 2, pp.833-843.

Gandomkar, M. and Tolabi, H. (2010) 'Investigation of simulated annealing, ant-colony and genetic algorithms for distribution network expansion planning with distributed generation', Proceedings of the 9th WSEAS Int. Conference on Instrumentation, Measurement, Circuits and Systems, pp.48-52.

Hadley, D.L. (1993) 'Daily variations in HVAC system electrical energy consumption in response to different weather conditions', Energy and Buildings, Vol. 19, No. 3, pp.235-247.

Independent Electricity System Operator (2015a) Feed-in-Tarif Program [online] http://www.ieso. ca/Pages/Participate/Markets-and-Programs/Procurement-Market.aspx.

Independent Electricity System Operator (2015b) Monthly Average Hourly Prices [online] http://www.ieso.ca/Pages/Power-Data/price.aspx.

Kalantari, M. and Kazemi, A. (2011) 'Placement of distributed generation unit and capacitor allocation in distribution systems using genetic algorithm', 10th International Conference on Environment and Electrical Engineering, May, No. 2, pp.1-5, IEEE.

Liang, H., Tamang, A.K., Zhuang, W. and Shen, X.S. (2014) 'Stochastic information management in smart grid', IEEE Communications Surveys \& Tutorials, Vol. 16, No. 3, pp.1746-1770.

Liew, S.N. and Strbac, G. (2002) 'Maximising penetration of wind generation in existing distribution networks', IEE Proceedings - Generation, Transmission and Distribution, May, Vol. 149, No. 3, p.256.

Marra, F., Fawzy, Y.T., Bulo, T. and Blažič, B. (2012) 'Energy storage options for voltage support in low-voltage grids with high penetration of photovoltaic', IEEE PES Innovative Smart Grid Technologies Conference Europe, pp.1-7.

Masters, G.M. (2013) Renewable and Efficient Electric Power Systems, John Wiley \& Sons.

Menniti, D., Merlo, M., Scordino, N. and Zanellini, F. (2012) 'Distribution network analysis: a comparison between hosting and loading capacities', SPEEDAM - 21st International Symposium on Power Electronics, Electrical Drives, Automation and Motion, pp.926-933.

Mori, H. and Yoshida, T. (2009) 'An efficient multi-objective memetic algorithm for uncertainties in distribution network expansion planning', IEEE Power \& Energy Society General Meeting, IEEE, July, No. 2, pp.1-6.

Mostafa, H.A., El-Shatshat, R. and Salama, M. (2013) 'Multi-objective optimization for the operation of an electric distribution system with a large number of single phase solar generators', IEEE Transactions on Smart Grid, Vol. 4, No. 2, pp.1038-1047. 
Pilo, F., Jupe, S., Silvestro, F., El Bakari, K., Abbey, C., Celli, G., Taylor, J., Baitch, A. and Carter-Brown, C. (2012) 'Planning and optimisation of active distribution systems - an overview of CIGRE Working Group C6.19 activities', CIRED Workshop: Integration of Renewables into the Distribution Grid, No. 135, pp.135-135, IET.

Reed, M.S., Graves, A., Dandy, N., Posthumus, H., Hubacek, K., Morris, J., Prell, C., Quinn, C.H. and Stringer, L.C. (2009) 'Who's in and why? A typology of stakeholder analysis methods for natural resource management', Journal of Environmental Management, Vol. 90, No. 5, pp.1933-1949.

Sheng, W., Liu, K., Liu, Y., Meng, X. and Li, Y. (2015) 'Optimal placement and sizing of distributed generation via an improved nondominated sorting genetic Algorithm II', IEEE Transactions on Power Delivery, Vol. 30, No. 2, pp.569-578.

Silvestri, A., Berizzi, A. and Buonanno, S. (1999) 'Distributed generation planning using genetic algorithms', PowerTech Budapest 99, Abstract Records (Cat. No.99EX376), p.257, IEEE, August.

Singh, D., Misra, R.K. and Singh, D. (2007) 'Effect of load models in distributed generation planning', IEEE Transactions on Power Systems, Vol. 22, No. 4, pp.2204-2212.

Xiang, L., Ng, D.W.K., Lee, W. and Schober, R. (2013) 'Optimal storage-aided wind generation integration considering ramping requirements', IEEE International Conference on Smart Grid Communications, SmartGridComm, pp.648-653.

You, S., Bindner, H.W., Hu, J. and Douglass, P.J. (2014) 'An overview of trends in distribution network planning: a movement towards smart planning', IEEE PES T\&D Conference and Exposition, IEEE, April, pp.1-5.

Zidan, A., Shaaban, M.F. and El-Saadany, E.F. (2013) 'Long-term multiobjective distribution network planning by DG allocation and feeders' reconfiguration', Electric Power Systems Research, Vol. 105, pp.95-104.

\section{Nomenclature}

\begin{tabular}{ll}
\hline \multicolumn{2}{l}{ Indices and sets } \\
\hline $\mathcal{D} \mathcal{G}$ & Set for all DG technologies available \\
$\mathcal{D}$ & Set of all days considered as representing a year \\
$\mathcal{G}$ & Set of grid connected busses $(\mathcal{G} \subseteq \mathcal{I})$ \\
$\mathcal{H}$ & Set of all hours considered as representing a day \\
$\mathcal{I}, \mathcal{J}$ & Set of all buses $\mathcal{I}=\mathcal{J}$ \\
$\mathcal{Q}_{d g}$ & Set of candidate buses for DG of technology $d g\left(\mathcal{Q}_{d g} \subseteq \mathcal{I}\right)$ \\
$\mathcal{Y}$ & Set of all years considered \\
$d$ & Index for days \\
$d g$ & Index for DG technology \\
$h$ & Index for hours \\
$i, j$ & Index for bus y index for years \\
\hline Parameters & \\
\hline$\theta_{(i, j, y)}$ & Angle of the Y-bus matrix admittance \\
$C_{\mathrm{UP}_{(l)}}$ & Cost of upgrading line $l$ in $\$$ \\
$I_{(l)}^{C A P}$ & Current carrying capacity of line $l$ \\
LH & Length of line $l$ \\
\hline
\end{tabular}




\section{Nomenclature (continued)}

\begin{tabular}{|c|c|}
\hline \multicolumn{2}{|l|}{ Parameters } \\
\hline$P F_{(i, d g, h, d, y)}$ & $\begin{array}{l}\text { Minimum allowed power factor for DG } d g \text { in bus } i \text { at hour } h \text {, day } d \text {, and year } y \\
(\$ / \mathrm{MW})\end{array}$ \\
\hline$r$ & Effective discount rate (discount and inflation) (Masters, 2013) \\
\hline$V^{\text {target }}$ & Targeted voltage level \\
\hline$Y_{(i, j, y)}$ & Magnitude of the Y-bus matrix admittance \\
\hline$C_{(\text {base })}^{\text {Unit }}$ & Base cost of upgrading lines per unit length \\
\hline$C_{(i, d g, h, d, y)}^{\mathrm{APower}}$ & Active power cost for DG $d g$ in bus $i$, at hour $h$, day $d$, and year $y(\$ / \mathrm{MW})$ \\
\hline$C_{(i, h, d, y)}^{\mathrm{APower}}$ & Active power cost from grid in bus $i$, at hour $h$, day $d$, and year $y(\$ / \mathrm{MW})$ \\
\hline$C_{(i, d g, y)}^{\text {Cap }}$ & $\begin{array}{l}\text { Capital cost of every allocated DG of technology } d g \text { at bus } i \text { and at year } y \\
\text { (\$MVA) }\end{array}$ \\
\hline$C_{(i, d g, h, d, y)}^{\mathrm{Fuel}}$ & Fuel cost for DG $d g$ in bus $i$, at hour $h$, day $d$, and year $y(\$ / \mathrm{MW})$ \\
\hline$C_{(i, d g, y)}^{\mathrm{OM}}$ & $\begin{array}{l}\text { Operation and maintenance cost for DG of technology } d g \text {, at bus } i \text { and year } y \\
(\$ / M W)\end{array}$ \\
\hline$C_{(i, d g, h, d, y)}^{\mathrm{RPower}}$ & Reactive power cost for DG $d g$ in bus $i$, at hour $h$, day $d$, and year $y$ (\$/MVAR) \\
\hline$C_{(i, h, d, y)}^{\mathrm{RPower}}$ & Reactive power cost from grid in bus $i$, at hour $h$, day $d$, and year $y$ (\$/MVAR) \\
\hline$C_{(i, d g, h, d, y)}^{\text {Unused }}$ & Cost of curtailed power for DG $d g$ in bus $i$, at hour $h$, day $d$, and year $y(\$ / \mathrm{MW})$ \\
\hline$P_{D(i, h, d, y)}$ & Active demand for bus $i$ at hour $h$, day $d$, and year $y$ \\
\hline$Q_{D(i, h, d, y)}$ & Reactive demand for bus $i$ at hour $h$, day $d$, and year $y$ \\
\hline$S_{d g}^{\max }$ & Maximum DG size of technology $d g$ \\
\hline$S_{i}^{\max }$ & Maximum allowed total DG capacity in bus $i$ \\
\hline$V_{\max }^{\text {spec }}$ & Specified maximum voltage \\
\hline$V_{\min }^{s p e c}$ & Specified minimum voltage \\
\hline$C_{(i, h, d, y)}^{\text {APower }}$ & Active power losses cost from in bus $i$, at hour $h$, day $d$, and year $y(\$ / \mathrm{MW})$ \\
\hline$C_{(i, h, d, y)_{\text {Loss }}}^{\mathrm{RPower}}$ & Reactive power losses cost from in bus $i$, at hour $h$, day $d$, and year $y$ (\$/MVAR) \\
\hline \multicolumn{2}{|l|}{ Acronyms } \\
\hline ANM & Active network management \\
\hline DER & Distributed energy resource \\
\hline DG & Distributed generation \\
\hline DISCO & Distribution company \\
\hline DNO & Distribution network operator \\
\hline DSM & Demand side management \\
\hline DSO & Distribution system operator \\
\hline IPP & Independent power provider \\
\hline LDC & Local distribution company \\
\hline NIST & National institute of standards and technology \\
\hline QoS & Quality of service \\
\hline SDS & Smart distribution system \\
\hline TVPP & Technical virtual power plant \\
\hline
\end{tabular}




\section{Nomenclature (continued)}

\begin{tabular}{|c|c|}
\hline Variables & \\
\hline$\delta_{(i, h, d, y)}$ & Angle of the voltage \\
\hline$\kappa_{(i, d g, y)}$ & Binary decision variables to place DG of technology $d g$ at bus $i$ and year $y$ \\
\hline $\mathrm{UP}_{(l)}$ & Binary decision to upgrade line 1 \\
\hline$\Omega$ & Vector of decision variables \\
\hline$\varpi_{(i, d g, y)}$ & Variable size of allocated DG of technology dg \\
\hline$A_{(l)}$ & A variable multiplier denoting the size of required line upgrade \\
\hline$I_{(l, h, d, y)}$ & Current in line $l$ at hour $h$, day $d$, and year $y$ \\
\hline$V_{(i, h, d, y)}$ & Magnitude of the voltage \\
\hline$\Psi_{(i, d g, h, d, y)}$ & $\begin{array}{l}\text { Decision variable for curtailed power in bus } i \text { at hour } h \text {, day } d \text {, and year } y \\
\left(\Psi_{(i, d g, h, d, y)} \in[0,1]\right)\end{array}$ \\
\hline$A F_{(d g, h, d, y)}$ & Availability factor for DG of technology $d g$ in bus $i$ at hour $h$, day $d$, and year $y$ \\
\hline$I_{(l, y) \max }$ & Maximum allowed current to flow in line $l$ at year $y$ \\
\hline$P_{(i, d g, h, d, y)}^{\text {Unused }}$ & Active power curtailed of DG $d g$ in bus $i$, at hour $h$, day $d$, and year $y(\mathrm{MW})$ \\
\hline$P_{g(i, h, d, y)}$ & Active power purchased from grid in bus $i$, at hour $h$, day $d$, and year $y(\mathrm{MW})$ \\
\hline$P_{(i, h, d, y)_{(l o s s)}}$ & $\begin{array}{l}\text { Active power loss measured at bus } i \text {, hour } h \text {, day } d \text {, and year } y \text { (difference } \\
\text { between produced power and demand) (MW) }\end{array}$ \\
\hline$P_{(i, d g, h, d, y)}$ & $\begin{array}{l}\text { Produced active power for DG of technology dg in bus } i \text {, at hour } h \text {, day } d \text {, and } \\
\text { year } y\end{array}$ \\
\hline$Q_{g(i, h, d, y)}$ & $\begin{array}{l}\text { Reactive power purchased from grid in bus } i \text {, at hour } h \text {, day } d \text {, and year } y \\
\text { (MVAR) }\end{array}$ \\
\hline$Q_{(i, h, d, y)(l o s s)}$ & $\begin{array}{l}\text { Reactive power loss measured at bus } i \text {, hour } h \text {, day } d \text {, and year } y \text { (difference } \\
\text { between produced power and demand) (MVAR) }\end{array}$ \\
\hline$Q_{(i, d g, h, d, y)}$ & $\begin{array}{l}\text { Produced reactive power for DG of technology dg in bus } i \text { at hour } h \text {, day } d \text {, and } \\
\text { year } y\end{array}$ \\
\hline$S_{(i, d g, y)}$ & Allocated DG of technology $d g$ at bus $i$ and year $y$ \\
\hline$y_{U P_{(l)}}$ & Year at which line $l$ is upgraded \\
\hline
\end{tabular}

